\title{
Shapes, multiple twins and surface structures of monodisperse FePt magnetic nanocrystals
}

\author{
Zu Rong Dai ${ }^{a}$, Shouheng Sun ${ }^{\mathrm{b}}$, Zhong L. Wang ${ }^{\mathrm{a}, *}$ \\ a School of Materials Science and Engineering, Georgia Institute of Technology, Atlanta, GA 30332-0245, USA \\ ${ }^{\mathrm{b}}$ IBM T.J. Watson Research Center, Yorktown Heights, NY 10598, USA
}

Received 22 October 2001; accepted for publication 14 January 2002

\begin{abstract}
Shapes, surface atomic arrangement and structural evolution induced by annealing of monodisperse FePt nanocrystals, synthesized by a solution phase chemical procedure, have been studied by high-resolution transmission electron microscopy. The as-synthesized FePt nanocrystals display dominantly a truncated octahedron shape enclosed by flat $\left\{\begin{array}{ll}0 & 0\end{array}\right\}$, stepped $\left\{\begin{array}{lll}1 & 1\end{array}\right\}$ and zig-zag $\left\{\begin{array}{lll}1 & 0\end{array}\right\}$ facets. The Marks decahedron FePt nanocrystals and the icosahedron based multiply twined FePt nanocrystals are identified in the as-synthesized particles. An improved structural model has been proposed for the multiply twined nanocrystals. After annealing, the $\left\{\begin{array}{lll}1 & 0\end{array}\right\}$ facets disappear and a regular cuboctahedron becomes the dominant shape. Surfaces of the FePt nanocrystals show no reconstruction but with some atomic steps and kinks. (C) 2002 Elsevier Science B.V. All rights reserved.
\end{abstract}

Keywords: Alloys; Electron microscopy; Polycrystalline surfaces

\section{Introduction}

The unique properties of nanoparticles are determined by their size, shape, crystal structure and surface structure [1]. Producing nanoparticles with controlled size and shape is important for nanotechnology. Nanoparticle shape not only determines the atomic arrangement on their surfaces but also plays an important role in controlling their assembly [2]. The structure and shape of nanoparticles such as metals [3-11], diamond

\footnotetext{
${ }^{*}$ Corresponding author. Tel.: +1-404-894-8008; fax: +1-404894-9140.

E-mail address: zhong.wang@mse.gatech.edu (Z.L. Wang).
}

$[12,13]$ and element semiconductors $[14,15]$ have been extensively studied experimentally and theoretically [16-24]. The most frequently observed shapes at the nanometer scale are cuboctahedron, truncated octahedron, icosahedron, regular decahedron, the Marks truncated decahedron, star and round pentagonal configurations [20]. The observation of tetrahedron shaped nanoparticles has also been reported for Pt nanocrystals [25].

The chemically ordered FePt alloy ( $\mathrm{L}_{\mathrm{o}}, \mathrm{P} 4 /$ $\mathrm{mmm}$ ) is a promising material for a new generation of ultrahigh density magnetic recording media $[26,27]$, since it has a large uniaxial magnetocrystalline anisotropy $\left(K_{\mathrm{u}} \cong 7 \times 10^{6} \mathrm{~J} / \mathrm{m}^{3}\right)[28,29]$ and shows excellent hard magnetic properties [30-33]. The fabrication of nano-size FePt particles with 
controlled size and composition is a key for its applications. Recently, a solution phase chemical procedure has been developed to synthesize monodispersive FePt nanocrystals with controlled size and composition [33]. X-ray diffraction and electron diffraction have shown that the as-synthesized $\mathrm{FePt}$ nanocrystals are of a chemically disordered face center cubic (fcc) lattice structure (A1, Fm $\overline{3} \mathrm{~m}$ ) $[33,34]$. A post-deposition annealing is needed to obtain the chemically ordered $\mathrm{L} 1_{\mathrm{o}}-\mathrm{FePt}$ phase. We have reported a detailed transmission electron microscopy (TEM) study about the annealing induced phase transformation and accompanying microstructure evolution, such as coalescence of FePt nanocrystals and formation of twin structure, for the $6 \mathrm{~nm} \mathrm{FePt}$ nanocrystals [34]. In this paper, we report our detailed study on atomic-scale structures of the as-synthesized and annealed FePt nanocrystals.

\section{Experimental methods}

Monodispersive FePt nanocrystals are synthesized by reduction of platinum acetylacetonate $\left(\mathrm{Pt}\left(\mathrm{CH}_{3} \mathrm{COCHCOCH}\right)_{2}\right)$ and decomposition of iron pentacarbonyl $\left(\mathrm{Fe}(\mathrm{CO})_{5}\right)$ in the presence of oleic acid and oleyl amine stabilizers [33]. The size and composition of the FePt nanocrystals can be readily controlled. Their composition is adjusted by controlling the molar ratio of the iron carbonyl to the platinum salt. In present study, the $6 \mathrm{~nm}$ FePt nanocrystals are synthesized by first growing $3 \mathrm{~nm}$ monodisperse FePt seed crystals, and then adding more reagents to enlarge the seed crystals to desired size under a 2:1 molar ratio of iron carbonyl to the platinum salt. These nanocrystals are isolated and purified by centrifugation after the addition of a flocculent (for example, ethanol) and can be re-dispersed in non-polar solvents in a variety of concentrations. TEM samples were prepared by depositing a droplet of the diluted FePt solution onto a carbon coated copper grid. Annealing was carried out in a Hitachi HF-2000 field emission transmission electron microscope by using a specimen heating holder. TEM observations were carried out at $200 \mathrm{kV}$ using the Hitachi HF-2000 and at $400 \mathrm{kV}$ using the JEOL-4000EX high-resolution transmission electron microscopy (HRTEM).

\section{Experimental results}

\subsection{Morphology and crystal structure}

The as-synthesized FePt nanocrystals have been examined by TEM. Fig. 1(a) is a low magnification TEM image showing a typical morphology of the product, where the monodisperse FePt nanocrystals with sizes of $\sim 6 \mathrm{~nm}$ in diameter self-assemble to form a two-dimensional distribution onto the supporting carbon film. A select-area electron diffraction pattern (Fig. 1(c)) indicates that the assynthesized FePt nanocrystals are of a fcc lattice structure with a lattice constant $a \cong 0.38 \mathrm{~nm}$, confirming the formation of the chemically disordered A1-FePt phase. The composition of the nanocrystals is determined to be $\mathrm{Fe}_{52} \mathrm{Pt}_{48}$ by energy dispersive X-ray spectroscopy, which is consistent with the expected composition.

To obtain the chemically ordered ferromagnetic $\mathrm{L}_{\mathrm{o}}-\mathrm{FePt}$ phase, in situ annealing was carried out in TEM. Shown in Fig. 1(b) is an TEM image of the FePt nanocrystals isothermally treated at $530{ }^{\circ} \mathrm{C}$ for $1 \mathrm{~h}$. The corresponding electron diffraction pattern is shown in Fig. 1(d), in which the extra reflection rings, such as $\left\{\begin{array}{lll}1 & 1 & 0\end{array}\right\}$, $\{120\},\left\{\begin{array}{lll}1 & 2\end{array}\right\}$, etc., appear, indicating the occurrence of phase transformation from the chemically disordered fcc A1 phase to the chemically ordered face centered tetragonal $\mathrm{L} 1$ o phase. The inset in Fig. 1(b) shows a TEM image taken from the specimen undergone an isothermal treatment at $600{ }^{\circ} \mathrm{C}$ for $1 \mathrm{~h}$, after which most of the nanocrystals had coalesced with only some nanocrystals in the monolayer region remain intact.

\subsection{Truncated octahedral FePt nanocrystals}

Detailed examination of the individual $\mathrm{FePt}$ nanocrystals shown in Fig. 1(a) and (b) indicates that both the as-synthesized and annealed FePt nanocrystals consist of certain facets, implying that the FePt nanocrystals are of polyhedral 

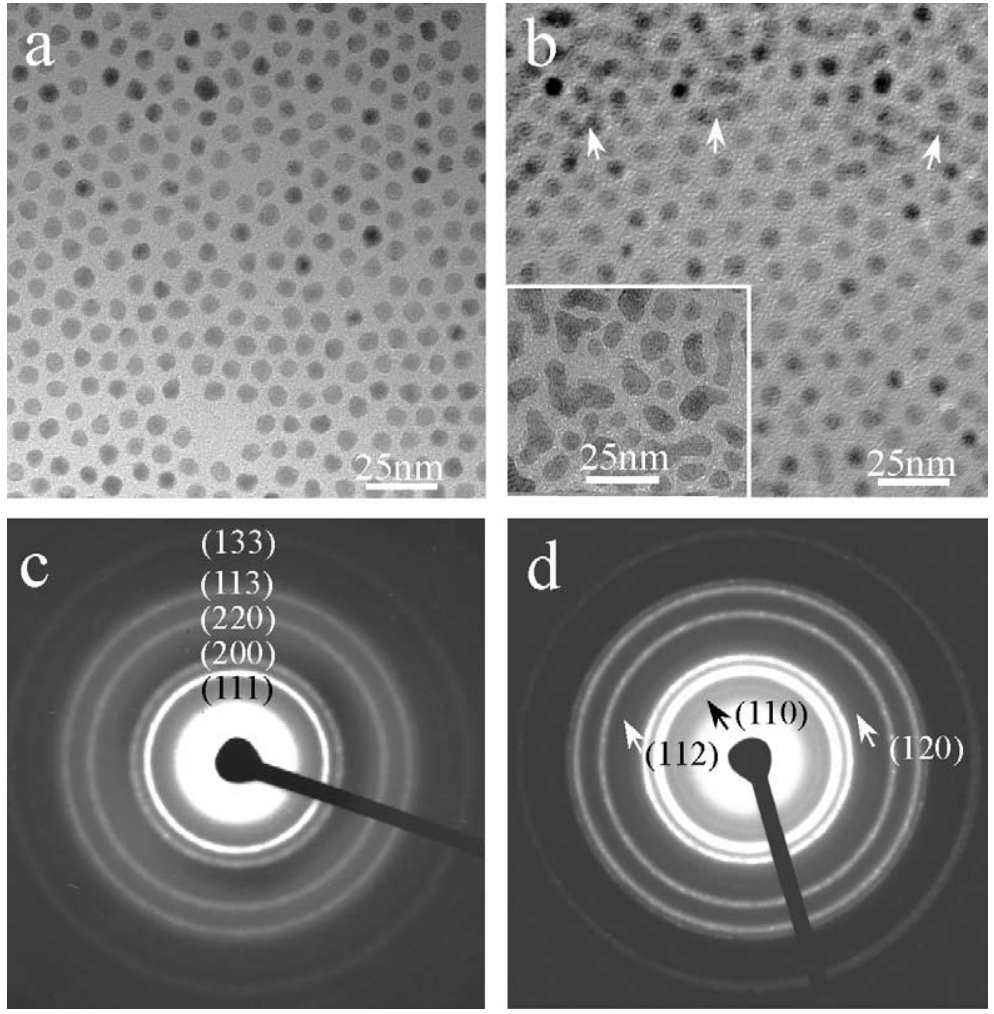

Fig. 1. TEM image of the as-synthesized FePt nanocrystals (a) and the corresponding select area electron diffraction pattern (c). (b) and (d) TEM images of the $\mathrm{Fe}_{52} \mathrm{Pt}_{48}$ nanocrystals after annealing at $530{ }^{\circ} \mathrm{C}$ for $1 \mathrm{~h}$ and the corresponding select area electron diffraction pattern, respectively. The inset in (b) is a TEM image of the nanocrystals post annealing at $600{ }^{\circ} \mathrm{C}$ for $1 \mathrm{~h}$.

shapes in three-dimension. The polyhedral shapes are studied by HRTEM. Fig. 2(a) and (b) are HRTEM images of two typical as-synthesized FePt nanocrystals projecting along [1 110$]$. Fig. 2(e) shows an HRTEM image of the $\mathrm{L}_{\mathrm{o}}-\mathrm{FePt}$ nanocrystal subjected to an annealing at $600{ }^{\circ} \mathrm{C}$ for $1 \mathrm{~h}$, in which electron beam is parallel to [1 10$]$ and the structural modulation, as marked by white arrowheads, is associated with chemical ordering in the crystal [34]. The annealed FePt nanocrystal has a projected shape of hexagon along its $\left[\begin{array}{lll}1 & 1 & 0\end{array}\right]$ direction, and is composed of flat $\left\{\begin{array}{lll}1 & 0 & 0\end{array}\right\}$ and $\{111\}$ planes. The polyhedral shape of the annealed FePt nanocrystals, therefore, is determined to be a cuboctahedron (a special case of truncated octahedron), as schematically depicted in Fig. 2(f), where the shadow plane corresponds to the projection plane of the HRTEM image given in Fig. 2(e). In contrast to the case of annealed FePt nanocrystals, the polyhedral shape of the as-synthesized FePt nanocrystals is enclosed by $\left\{\begin{array}{lll}1 & 0 & 0\end{array}\right\}$, $\{111\}$ and $\{110\}$ crystallographic planes and their surfaces are not flat at atomic scale. Some surface defects such as atomic steps and kinks are identified, as indicated by white arrowheads in Fig. 2(a) and (b). The details of atomic arrangements on the surfaces of FePt nanocrystals will be described in Section 3.4. Schematic diagrams of the polyhedra corresponding to the FePt nanocrystals shown in Fig. 2(a) and (b) are given in Fig. 2(c) and (d), respectively, and the projecting planes corresponding to the HRTEM images are represented by shadowed planes. Evidently, the shape of as-synthesized FePt nanocrystals is truncated octahedron with the surfaces not as flat as those for annealed FePt nanocrystals. 

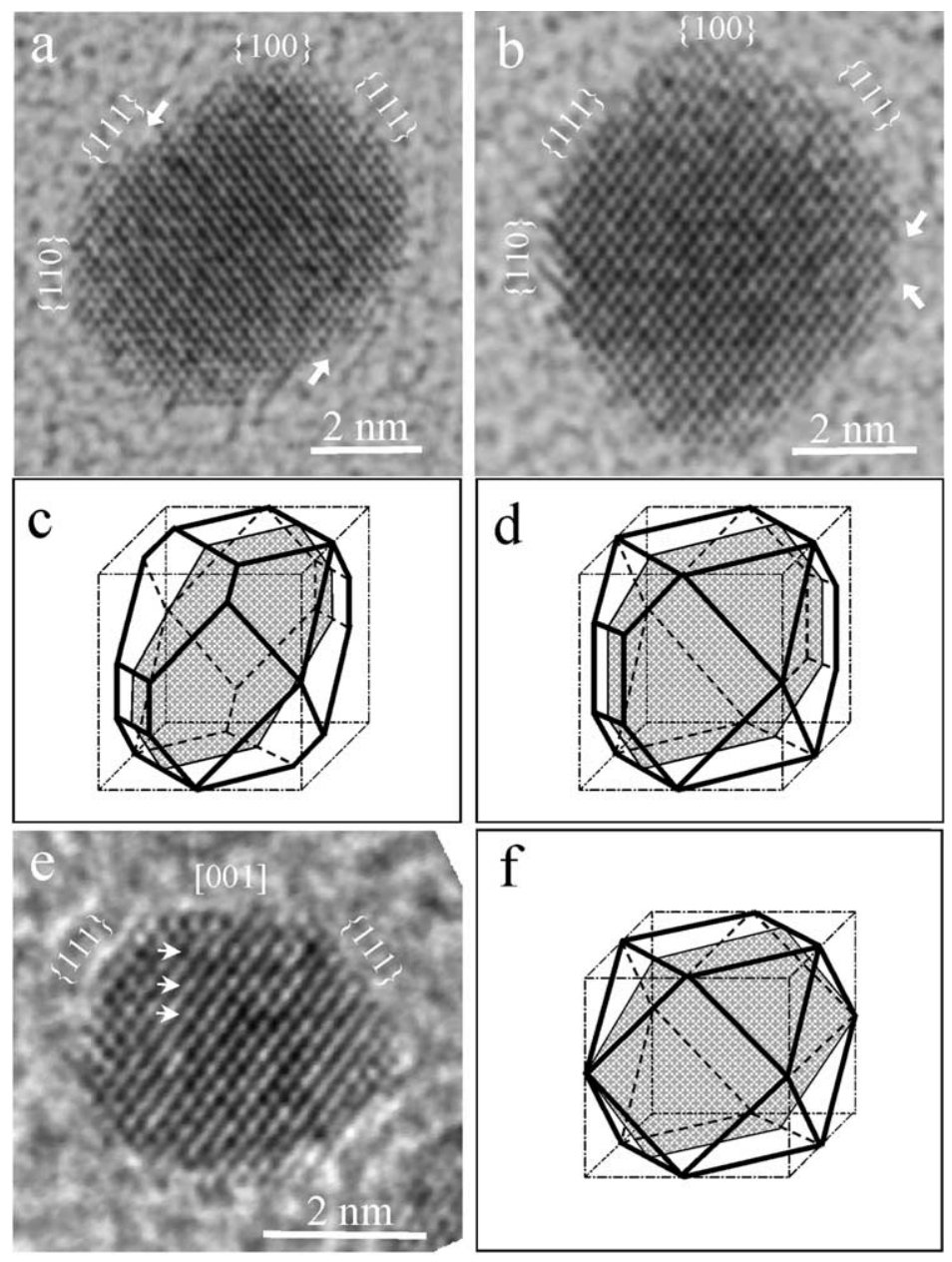

Fig. 2. (a) and (b) HRTEM images of individual FePt nanocrystals prior annealing. (c) and (d) Schematic models of the truncated octahedra. (e) HRTEM image of a FePt nanocrystal after annealing at $530{ }^{\circ} \mathrm{C}$ for $1 \mathrm{~h}$. (f) A corresponding cuboctahedron shape.

\subsection{Multiply twined FePt nanocrystals}

Although the predominant shape for both the as-synthesized and the annealed FePt nanocrystals is truncated octahedron, some other shapes are also observed. Fig. 3(a) shows an HRTEM image of the as-synthesized FePt nanocrystal, in which the five-fold twinning related domains are identified. In order to study the structure in detail, image processing is demonstrated by the Fourier-filtering technique [35]. Simply, a fast Fourier transform (FFT) is applied to a digitized HRTEM image for producing a power spectrum of the image. Then
Bragg filter is generated with circular "holes" at the positions of the Bragg spots of the periodic part of the image. The multiplication of the power spectrum with the filter is followed by a reverse FFT to produce a filtered image. A proper choice of the size of the holes is important to prevent artifacts in the image. Fig. 3(d) is a FFT of the image shown in Fig. 3(a), and Fig. 3(b) is a corresponding filtered image. The dotted lines marked by $\mathrm{OA}, \mathrm{OB}, \mathrm{OC}, \mathrm{OD}$, and $\mathrm{OE}$ in Fig. 3(b) represent the positions of the twin boundaries. The twin boundaries are coherent and free from defects such as stacking fault, dislocation or micro-twin. The 


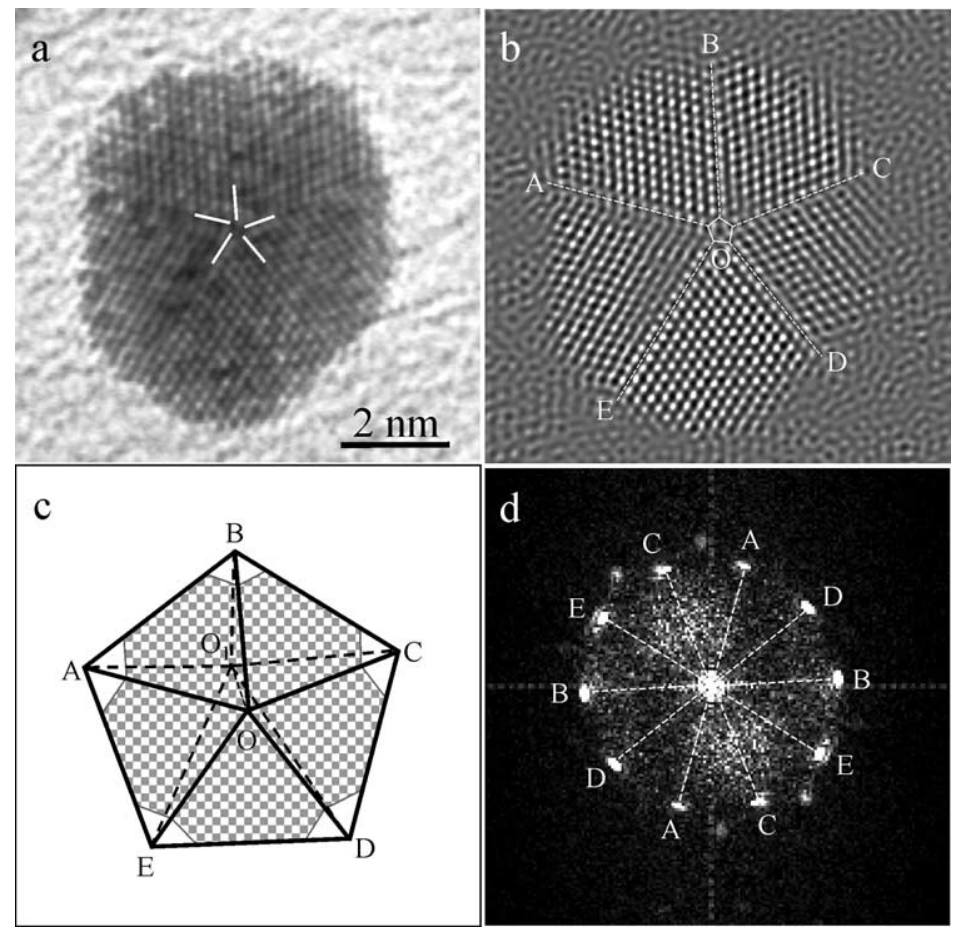

Fig. 3. (a) HRTEM image of the as-synthesized FePt nanocrystal with the Marks decahedron shape. (b) Fourier filtered image. (c) Schematic polyhedron model. (d) FFT pattern of the image shown in (a).

angle between two adjacent boundaries varies within $71.5-72.5^{\circ}$. This feature can also be verified from the FFT pattern (Fig. 3(d)), where reflections $\mathrm{A}, \mathrm{B}, \mathrm{C}, \mathrm{D}$, and $\mathrm{E}$ are produced from the twin boundaries $\mathrm{OA}, \mathrm{OB}, \mathrm{OC}, \mathrm{OD}$, and $\mathrm{OE}$, respectively. The shape of the nanocrystal obviously is the so-called Marks decahedron [7], i.e., a truncated regular decahedron. Fig. 3(c) gives schematic diagrams of a regular decahedron $\mathrm{OO}_{1}-\mathrm{ABCD}$, and the Marks decahedron is shown in shadow.

Shown in Fig. 4(a) is another FePt nanocrystal with a spherical shape. The corresponding Fourier filtered image (Fig. 4(b)) and FFT pattern (Fig. 4(d)), however, illustrate that the nanocrystal consists of six domains with six-fold twins. The twin boundaries are indicated by dashed lines OF, OP, OL, OR, OI and OQ, as shown in Fig. 4(b), in which neither the twin boundary OF is parallel to the twin boundary OR, nor OQ to OL. The angles between $\mathrm{OF}$ and $\mathrm{OR}$, and $\mathrm{OQ}$ and $\mathrm{OL}$ are about $6^{\circ}$, which is responsible for the split spots $\mathrm{K}$ and $\mathrm{J}$,

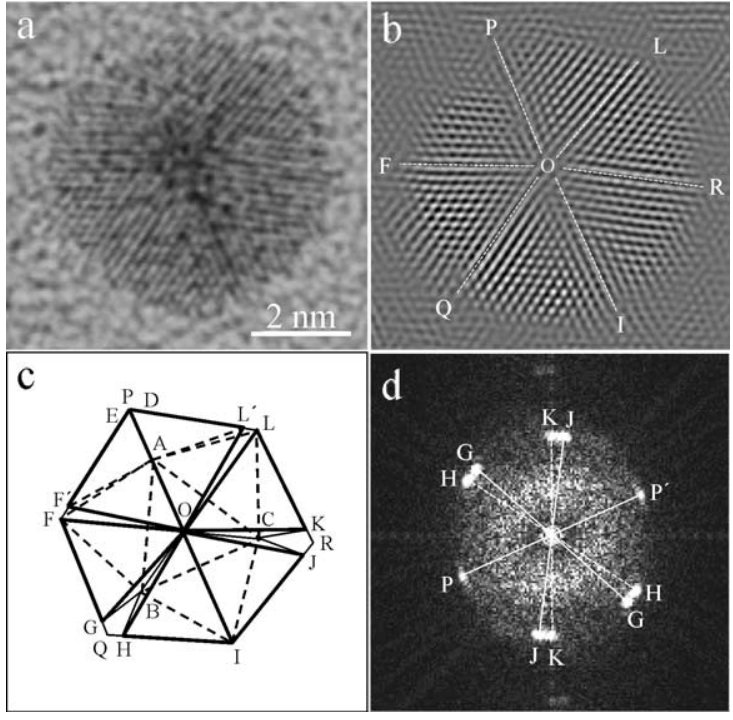

Fig. 4. (a) HRTEM image of the as-synthesized FePt nanocrystal with icosahedron related shape. (b) Fourier filtered image. (c) Schematic polyhedron model. (d) FFT pattern of the image shown in (a). 
and $\mathrm{H}$ and $\mathrm{G}$ in the FFT pattern (Fig. 4(d)). In contrast, the twin boundary OI is parallel to that of OP. The corresponding reflections $\mathrm{P}$ and $\mathrm{P}^{\prime}$ in Fig. 4(d), therefore, are not split although they are a bit diffuse. Fig. 4(c) gives a schematic model of the twins in the FePt nanocrystal and it will be discussed in detail in Section 4.1.

\subsection{Surface structure of FePt nanocrystals}

Prior to annealing, the predominant shape of the as-synthesized FePt nanocrystals is truncated octahedron enclosed by $\left\{\begin{array}{llll}1 & 1 & 1\end{array}\right\},\left\{\begin{array}{lll}1 & 0 & 0\end{array}\right\}$ and $\left\{\begin{array}{lll}1 & 1 & 0\end{array}\right\}$ crystallographic planes. The $\left\{\begin{array}{lll}1 & 0 & 0\end{array}\right\}$ and $\left\{\begin{array}{lll}1 & 1 & 1\end{array}\right\}$ surfaces are relatively flat at atomic scale, as shown in Figs. 2(a), (b) and 5(a), (b). Some atomic steps are identified on the $\{111\}$ surfaces, as marked by arrowheads in Fig. 5(a) and (b). The $\{110\}$ surface, however, is rough and its projection along [ 1110$]$ displays zig-zag shape. Fig. 5(c) and (d) are two enlarged HRTEM images of the typical $\left\{\begin{array}{llll}1 & 1 & 0\end{array}\right\}$ surfaces, and Fig. 5(e) and (f) give schematic diagrams of atomic arrangement on the corresponding surfaces. It is evident that the zigzag surfaces consist of small $\left\{\begin{array}{lll}1 & 1 & 1\end{array}\right\}$ facets, resulting in the formation of kinks and twists on the $\left\{\begin{array}{lll}1 & 1 & 0\end{array}\right\}$ surfaces. This is the so-called missing-row reconstruction for lowering the surface energy.

After annealing, the $\{110\}$ surface disappears and the FePt nanocrystal is enclosed only by the $\left\{\begin{array}{lll}0 & 0\end{array}\right\}$ and $\left\{\begin{array}{lll}1 & 1\end{array}\right\}$ crystallographic planes, forming a regular cuboctahedron shape (Fig. 6(a) and 2(e)). All of the $\left\{\begin{array}{lll}1 & 0 & 0\end{array}\right\}$ and $\left\{\begin{array}{lll}1 & 1 & 1\end{array}\right\}$ surfaces are flat on atomic scale. Some lattice distortion is identified on the $\{100\}$ surface, as indicated by the dashed line on the HRTEM image (Fig. 6(a)), where the white line frame denotes a unit cell of the FePt crystal. Atomic arrangement on the $\left\{\begin{array}{lll}1 & 1\end{array}\right\}$ surface is relatively perfect (Fig. 6(a) and (d)) except at the corner where two different $\left\{\begin{array}{lll}1 & 1 & 1\end{array}\right\}$ planes intersect, and no surface reconstruction is identified. At the intersecting corner, atomic steps with a mono-atom height are identified, as shown in Fig. 6(d) and (e).

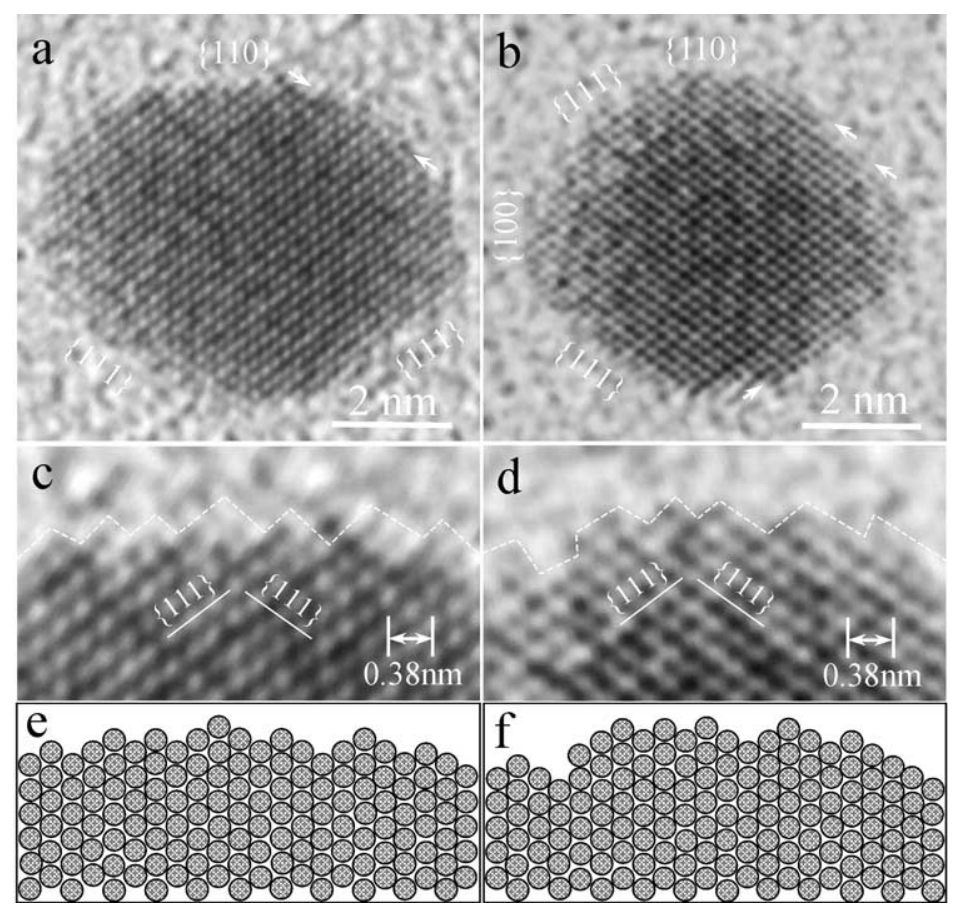

Fig. 5. (a) HRTEM images of the as-synthesized FePt nanocrystals. (c) and (d) Enlarged images of the $\{110\}$ faceted surfaces. (e) and (f) Schematic models of atomic arrangement on the $\left\{\begin{array}{lll}1 & 0 & 0\end{array}\right\}$ surfaces. 


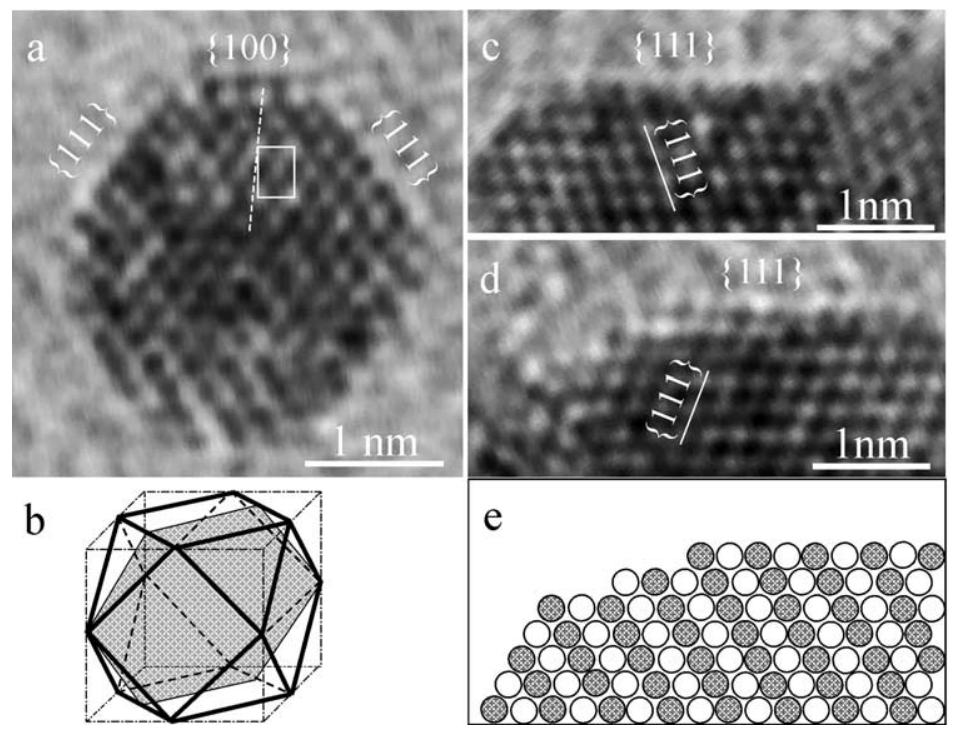

Fig. 6. (a) HRTEM image of an FePt nanocrystal post annealed at $530{ }^{\circ} \mathrm{C}$ for $1 \mathrm{~h}$. (b) Schematic model of a cuboctahedron enclosed by $\left\{\begin{array}{lll}1 & 1 & 1\end{array}\right\}$ and $\left\{\begin{array}{lll}1 & 0 & 0\end{array}\right\}$ planes. (c) and (d) HRTEM images showing the corners where the two $\left\{\begin{array}{llll}1 & 1\end{array}\right\}$ crystal planes intersect. (e) Schematic model of atomic arrangement.

\section{Discussion}

\subsection{Structure of multiply twined nanocrystals}

Multiply twined particles are usually observed in fcc structured small metal particle systems, which are mainly associated with either decahedron or icosahedron. The decahedron can be regarded as being composed of five tetrahedra $\mathrm{A}$ $\mathrm{EOO}_{1}, \mathrm{~B}-\mathrm{AOO}_{1}, \mathrm{C}-\mathrm{BOO}_{1}, \mathrm{D}-\mathrm{COO}_{1}$ and $\mathrm{E}-\mathrm{AOO}{ }_{1}$ by sharing faces, as shown in Fig. 3(c). For the case of fcc stacking, a tetrahedron is enclosed by four $\left\{\begin{array}{lll}1 & 1 & 1\end{array}\right\}$ crystal planes with an including angle of $70^{\circ} 32^{\prime}$ between two neighbor planes. If five fcc stacked tetrahedra directly form a decahedron, an $7^{\circ} 20^{\prime}$ gap remains. The gap can be accommodated by straining the atomic lattice. If the strain is uniformly distributed in the decahedron, a disclination will form at the center of the decahedral particle [36]. That is just the case of the FePt nanocrystal shown in Fig. 3. The strain can also be relaxed by forming stacking fault or micro-twin in the particle [20]. Several variants of the decahedron exist, such as star, round pentagonal, and Marks decahedron. Among those the Marks decahedron is the most common shape since it contains extra $\left\{\begin{array}{llll}1 & 1 & 1\end{array}\right\}$ facets, resulting in remarkable stability [20].

Ino [3] and Allpress and Sanders [5] first proposed several models for the multiply twined particles with shapes associated with icosahedron. Shown in Fig. 7(a) is one of the models. The multiply twined particle is formed by nucleation of a tetrahedral atomic cluster $\mathrm{O}-\mathrm{ABC}$ with its $\mathrm{ABC}$ plane facing on a substrate. Then three more tetrahedra F-AOB, I-BOC and L-AOC grow on three $\left\{\begin{array}{lll}1 & 1 & 1\end{array}\right\}$ planes of the nucleus $\mathrm{AOB}, \mathrm{BOC}$ and AOC by sharing a face, respectively, forming primary twins. Following that, six tetrahedra E-FAO, G-FBO, H-BIO, J-CIO, D-ALO and K-CLO grow on the surfaces of the primary twins to form secondary twins. The corresponding diffraction pattern is schematically shown in Fig. 7(c), where only the $\left\{\begin{array}{lll}1 & 1 & 1\end{array}\right\}$ reflections from the secondary twins are displayed. Comparing the diffraction pattern (Fig. 7(c)) with the FFT pattern (Fig. 4(d)), both are not matching because the reflections $\mathrm{P}$ and $\mathrm{P}^{\prime}$ in Fig. 4(d) are not splitting, while that occurs in Fig. 7(c). This indicates that the model (Fig. 7(a)) can not be used to explain the structure of the multiply twined nanocrystal displayed in Fig. 4. A modified model, therefore, is suggested, as shown in 


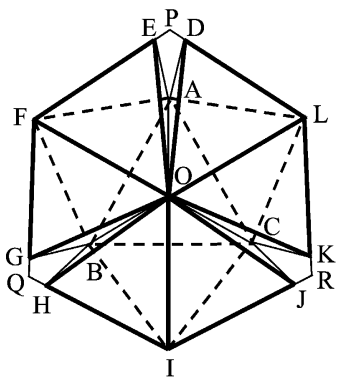

(a)

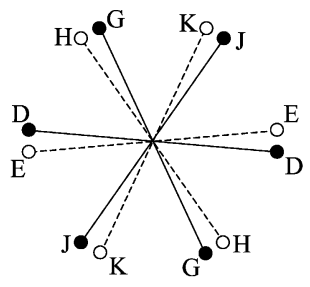

(c)

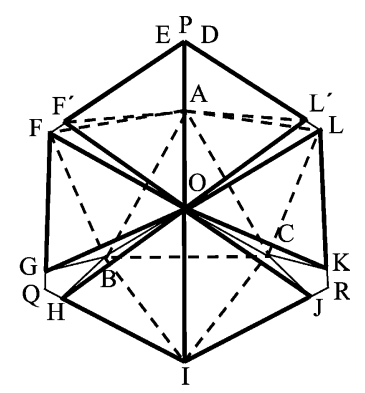

(b)

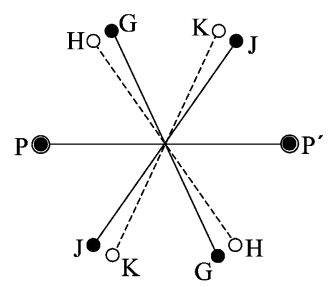

(d)
Fig. 7. The Ino's model of icosahedron related multiply twined nanoparticle (a) and corresponding diffraction pattern (c). A new model suggested in present study (b) and corresponding diffraction pattern (d).

Fig. 7(b). The only difference between the modified model and the previous model (Fig. 7(a)) is that two $\left\{\begin{array}{lll}1 & 1 & 1\end{array}\right.$ planes DAO and FAO are joined in parallel. As a result, the split reflections $E$ and D (Fig. 7(c)) overlap together to form a reflection $\mathrm{P}$, which reproduces the pattern of Fig. 4(d).

\subsection{Shape evolution of FePt nanocrystals by annealing}

The shape of the crystalline particles depends on not only thermal dynamics but also on kinetics. The faceting is the most favorable mechanism for minimizing particle surface energy. The preparation conditions and techniques, however, significantly influence the shape of the particles [37,38]. At nanometer scale, the most frequently observed shapes are truncated octahedron, cuboctahdron, icosahedron, decahedron and truncated decahedra including star, the round pentagon and the Marks decahedron. Although theoretical calculations indicate that the stability is different for different shapes [20], the shape usually is not unique for nanocrystals under the same conditions because of small differences in total energy among the different shapes.

Co-existing with the Marks decahedron and the icosahedron related multiply twined $\mathrm{FePt}$ nanocrystals, the truncated octahedron is identified to be the dominant shape for the as-synthesized FePt nanocrystals in present study. The Marks decahedron structure was first reported for Au particles and suggested to be a minimum energy configuration [18]. After annealing, the shapes of the uncoalesced FePt nanocrystals are dominated by the conventional cuboctahedron that is the most stable shape according to molecular dynamics simulation [20]. Multiply twined FePt nanocrystals associated with either decahedron or icosahedron are rarely observed after annealing, indicating that such sorts of multiple twins tend to disappear during annealing. As evidence, Fig. 8 shows an HRTEM image of a coalesced FePt nanocrystal that consists of two originally separated nanocrystals (grains), in which the grain boundary can still be distinguished, as marked by the white arrowheads. The white open arrowheads labeled on the grain on the right-hand side show compositional modulation related to the chemical ordering due to annealing [34]. The grain on the left-hand side is originally a decahedron shaped FePt nanocrystal. Its central axis has been displaced to a position close to an edge of the nanocrystal after annealing because of stress release. Occurrence of the chemical ordering might be another reason

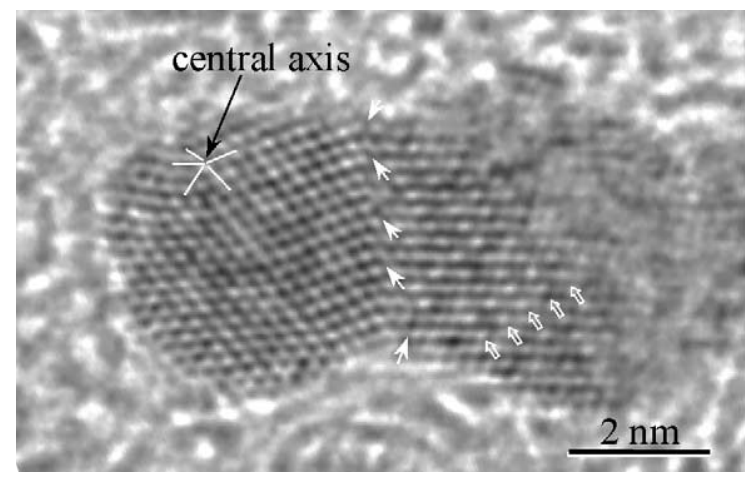

Fig. 8. Coalesced FePt nanocrystal consisting of two original FePt nanocrystals, and one of them is a decahedron. 
that results in the decay of the multiply twined FePt nanocrystals. Instead of forming the multiple twins related decahedron and icosahedron, another type of multiple twins is observed in the coalesced FePt nanocrystals after annealing, as shown in Fig. 9(b), where the twin boundaries are indicated by white dashed lines. Formation of this type of twin structure is believed to be associated with the chemical ordering created by annealing [34]. Similar twin structure is also observed in the as-synthesized FePt nanocrystals with chemically disordered A1 crystal structure. As a difference from the twin structure in coalesced FePt nanocrystals, however, only single twin boundary has

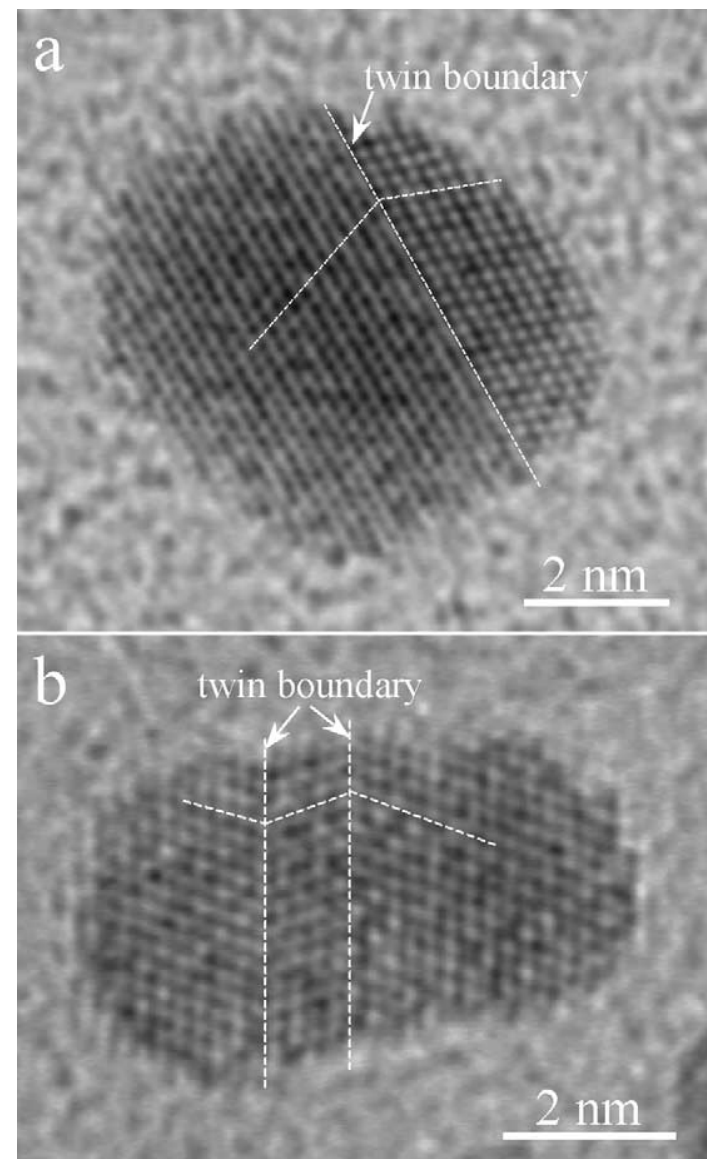

Fig. 9. (a) HRTEM image of an as-synthesized FePt nanocrystal with a twin structure. (b) HRTEM image of a coalesced FePt nanocrystal post annealed at $600{ }^{\circ} \mathrm{C}$ for $1 \mathrm{~h}$, which includes two twin boundaries. been observed in the as-synthesized FePt nanocrystals (Fig. 9(a)).

\subsection{Atomic arrangement on FePt nanocrystals surfaces}

The as-synthesized FePt nanocrystals have the shape of a truncated octahedron enclosed by

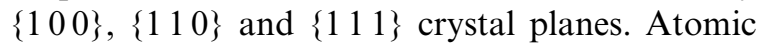
arrangements on the $\{100\}$ and $\{111\}$ surfaces are basically the same as those of corresponding crystal planes in the volume although the atomic arrangements are not flat across the surfaces due to presence of atomic steps. The $\{110\}$ surface is rough on atomic scale and displays zig-zag consisting of $\left\{\begin{array}{lll}1 & 1\end{array}\right\}$ facets. During annealing, while the A1-FePt phase transforms into the chemically ordered $\mathrm{L1}_{\mathrm{o}}-\mathrm{FePt}$ phase, $\left\{\begin{array}{lll}1 & 1 & 0\end{array}\right\}$ surfaces tend to disappear, and $\left\{\begin{array}{lll}0 & 0\end{array}\right\}$ and $\left\{\begin{array}{lll}1 & 1 & 1\end{array}\right\}$ surfaces evolve. After annealing, the uncoalesced FePt nanocrystals remain in the shape of cuboctahedron enclosed only by flat $\{100\}$ and $\{111\}$ surfaces. Atomic arrangements on the $\left\{\begin{array}{lll}1 & 0 & 0\end{array}\right\}$ and $\left\{\begin{array}{lll}1 & 1 & 1\end{array}\right\}$ surfaces are quite regular. The surfaces of the coalesced FePt nanocrystals also consist of $\left\{\begin{array}{lll}1 & 0 & 0\end{array}\right\}$ and $\left\{\begin{array}{lll}1 & 1 & 1\end{array}\right\}$ planes although the surfaces may not be flat. Fig. 10(a) shows an HRTEM image corresponding to a part of the surface of a coalesced FePt nanocrystal. It is evident that the surface consists of $\left(\begin{array}{lll}0 & 0 & 1\end{array}\right)$ and $\left\{\begin{array}{lll}1 & 1 & 1\end{array}\right\}$ facets. An alternative change in intensity of the image spots along the vertical direction is clearly identified from the HRTEM image (Fig. 10(a)), which is associated with compositional modulation in the chemically ordered $\mathrm{L1}_{\mathrm{o}}-\mathrm{FePt}$ phase. The open arrowheads marked in Fig. 10(a) indicate the rows of the image spots with stronger intensity. Fig. 10(b) is a schematic atomic model extracted from the experimental HRTEM image (Fig. 10(a)). According to the model, it seems that the top layer of the $\left(\begin{array}{lll}0 & 0 & 1\end{array}\right)$ surface prefers to be occupied by one species of atoms ( $\mathrm{Fe}$ or $\mathrm{Pt})$. The inset in Fig. 10(a) is a simulated HRTEM image corresponding to the area of the crystal within the frame marked in Fig. 10(b). The image simulation has been done based on the dynamic electron diffraction theory under the conditions of: acceleration voltage $=400$ $\mathrm{kV}$, spherical coefficient $C_{\mathrm{s}}=1.0 \mathrm{~mm}$, specimen 


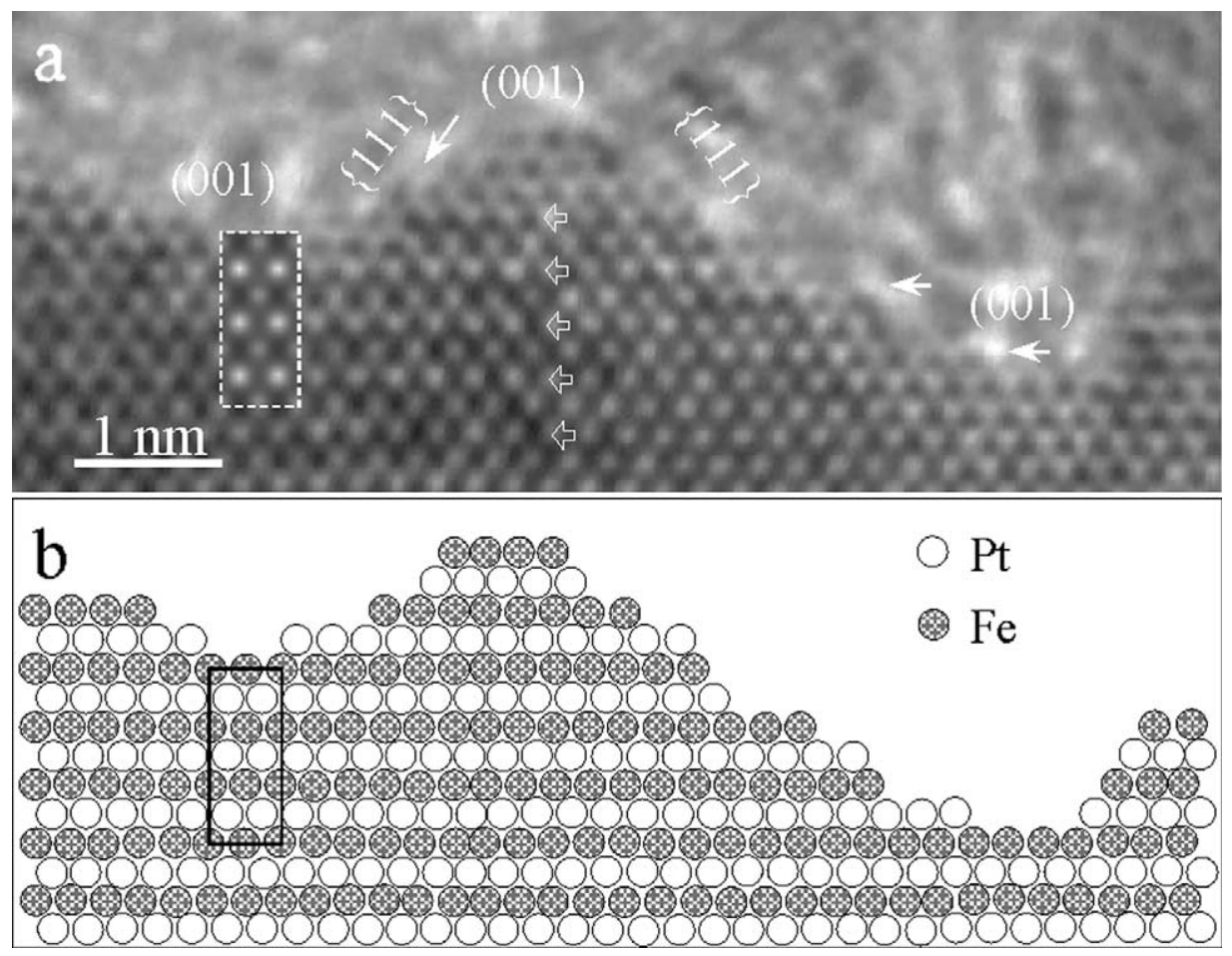

Fig. 10. (a) HRTEM image of a portion of the surface of a coalesced FePt nanocrystal post annealed at $600{ }^{\circ} \mathrm{C}$ for $1 \mathrm{~h}$. The inset is a simulated HRTEM image. (b) A schematic model for the atomic arrangement on the nanocrystal surface.

thickness $=8 \mathrm{~nm}$, defocus $=-74 \mathrm{~nm}$ and beam divergence $=0.15 \mathrm{mrad}$. In the simulated image, the spots with the strongest intensity correspond to the positions of the $\mathrm{Pt}$ atomic columns and the second strongest ones are the positions of the $\mathrm{Fe}$ atomic columns. The simulation image matches the experimental image very well even for the top layer, indicating the atomic arrangement in the top layer of the $(001)$ surface is almost the same as that in the volume. Based on the image simulationabove, it seems plausible that the $\left(\begin{array}{lll}0 & 0 & 1\end{array}\right)$ surface terminates with $\mathrm{Fe}$ atoms. Some further work, however, still is needed to unambiguously confirm it.

\section{Conclusions}

In conclusion, our HRTEM study of the FePt nanocrystals shows that the truncated octahedron, enclosed by flat $\left\{\begin{array}{lll}1 & 0 & 0\end{array}\right\}$, stepped $\left\{\begin{array}{lll}1 & 1 & 1\end{array}\right\}$ and zig-zag
$\{110\}$ facets, is the dominant shape for the assynthesized nanocrystals. The Marks decahedron FePt nanocrystals and icosahedron related multiply twined FePt nanocrystals are also identified in the as-synthesized nanocrystals. Based on experimental data, an improved structural model for the multiply twined nanocrystals based on icosahedron has been proposed. After annealing, the $\left\{\begin{array}{lll}1 & 1 & 0\end{array}\right\}$ facet disappears; the regular cuboctahedron becomes a dominant shape for the chemically ordered FePt nanocrystals, and the (0 0101$)$ surface prefers to be occupied by one type of atoms.

\section{Acknowledgements}

Thanks to the financial support from the US NSF grant DMR-9733160, DARPA(DAAD1901-1-0546), and the Georgia Tech Electron Microscopy Center for providing the research facility. 


\section{References}

[1] Z.L. Wang, J. Phys. Chem. B 104 (2000) 1153.

[2] Z.L. Wang, Z.R. Dai, S. Sun, Adv. Mater. 12 (2000) 1944.

[3] S. Ino, J. Phys. Soc. Jpn. 21 (1966) 346.

[4] S. Ino, S. Ogawa, J. Phys. Soc. Jpn. 22 (1967) 1365.

[5] J.G. Allpress, J.V. Sanders, Surf. Sci. 7 (1967) 1.

[6] L.D. Marks, D.J. Smith, J. Cryst. Growth 54 (1981) 425.

[7] L.D. Marks, Phil. Mag. 49 (1984) 81.

[8] L.D. Marks, Surf. Sci. 150 (1985) 385.

[9] N.J. Long, R.F. Marzke, M. Mckelvy, W.S. Glaunsinger, Ultramicroscopy 20 (1986) 15.

[10] S. Giorgio, J. Urban, Appl. Phys. Lett. 52 (1988) 1467.

[11] S. Giorgio, J. Urban, W. Kunath, Phil. Mag. A 60 (1989) 553.

[12] S. Matsumoto, Y. Masui, J. Mat. Sci. 18 (1983) 1785.

[13] A.R. Badzian, T. Badzian, R. Roy, R. Messier, K.E. Spear, Mat. Res. Bull. 23 (1988) 531.

[14] Y.S. Staito, K. Yatsuya, K. Mihama, R. Uyeda, Jpn. J. Appl. Phys. 17 (1979) 1149.

[15] S. Iijima, Jpn. J. Appl. Phys. 26 (1987) 365.

[16] P.M. Ajayan, L.D. Marks, Phase Transitions 24-26 (1990) 229.

[17] R. Uyeda, Prog. Mat. Sci. 35 (1991) 1.

[18] L.D. Marks, Rep. Prog. Phys. 57 (1994) 603.

[19] J.A. Ascencio, C. Gutiérrez-Wing, M.E. Espinosa, M. Marín, S. Tehuacanero, C. Zorrilla, M. José-Yacamán, Surf. Sci. 396 (1998) 349.

[20] M. José-Yacamán, J.A. Acencio, H.B. Liu, J. GardeaTorresdey, J. Vac. Sci. Technol. B 19 (2001) 1091.
[21] D.J. Wales, H.A. Scheraga, Science 285 (1999) 1368.

[22] J.P.K. Doye, D.J. Wales, Phys. Rev. Lett. 86 (2001) 5719.

[23] G. Bravo-Pérez, I.L. Garzón, O. Novaro, J. Mol. Struc. Theochem. 493 (1999) 225.

[24] H. Häkkinen, U. Landman, Phys. Rev. B 62 (2000) R2287.

[25] Z.L. Wang, T.S. Ahmad, M.A. Ei-Sayed, Surf. Sci. 380 (1997) 302.

[26] D. Weller, A. Moser, IEEE Trans. Magn. 35 (1999) 4423.

[27] J.A. Christodoulides, Y. Huang, Y. Zhang, G.C. Hadjipanayis, I. Panagiotopoulos, D. Niarchos, J. Appl. Phys. 87 (2000) 6938.

[28] O.A. Ivanov, L.V. Solina, V.A. Demshina, L.M. Magat, Phys. Met. Metallog. 35 (1973) 81.

[29] K. Inomata, T. Sawa, S. Hashimoto, J. Appl. Phys. 64 (1988) 2537.

[30] K. Watanabe, H. Masumoto, Trans. Jpn. Inst. Met. 9 (1983) 627.

[31] J.H. Westbrook, in: Intermetallic Compounds, John Wiley \& Sons Inc., New York, 1967, p. 541.

[32] K. Watanabe, H. Masumoto, Trans. Jpn. Inst. Met. 26 (1985) 362.

[33] S. Sun, C.B. Murray, D. Weller, L. Folks, A. Moser, Science 287 (2000) 1989.

[34] Z.R. Dai, S. Sun, Z.L. Wang, Nano Lett. 1 (2001) 443.

[35] A.F. de Jong, W. Coene, D. van Dyck, Ultramicroscopy 27 (1989) 53.

[36] R. De Wit, J. Phys. C: Solid State Phys. 5 (1972) 529.

[37] T. Wang, C. Lee, L.D. Schmidt, Surf. Sci. 163 (1985) 181.

[38] C.R. Henry, C. Chapon, C. Duriez, S. Giorgio, Surf. Sci. 253 (1991) 177. 